\title{
Three-Dimensional Single-Sided Marchenko Inverse Scattering, Data-Driven Focusing, Green's Function Retrieval, and their Mutual Relations
}

\author{
Kees Wapenaar, ${ }^{1, *}$ Filippo Broggini, ${ }^{2}$ Evert Slob, ${ }^{1}$ and Roel Snieder ${ }^{2}$ \\ ${ }^{1}$ Department of Geoscience and Engineering, Delft University of Technology, 2600 GA Delft, The Netherlands \\ ${ }^{2}$ Center for Wave Phenomena, Colorado School of Mines, Golden, Colorado 80401, USA
}

(Received 2 September 2012; published 22 February 2013)

\begin{abstract}
The one-dimensional Marchenko equation forms the basis for inverse scattering problems in which the scattering object is accessible from one side only. Here we derive a three-dimensional (3D) Marchenko equation which relates the single-sided reflection response of a 3D inhomogeneous medium to a field inside the medium. We show that this equation is solved by a 3D iterative data-driven focusing method, which yields the 3D Green's function with its virtual source inside the medium. The 3D single-sided Marchenko equation and its iterative solution method form the basis for imaging of 3D strongly scattering inhomogeneous media that are accessible from one side only.
\end{abstract}

DOI: 10.1103/PhysRevLett.110.084301

Introduction.-Inverse scattering is the problem of determining a medium perturbation (or, in quantum mechanics, a potential) from an acoustic, electromagnetic, or quantummechanical wave field, scattered by this perturbation (or potential). One particular approach to one-dimensional (1D) inverse scattering makes use of the Marchenko equation. This is an exact integral equation which relates the reflection response, measured at one side of the perturbation, to the field inside the medium [1,2]. Rose [3] shows that solving the Marchenko equation is equivalent to designing a wave field which, when emitted from one side of the perturbation into the inhomogeneous medium, focuses inside the medium. He proposes an iterative scheme to design the focusing wave field, which only requires the reflection response at one side of the perturbation as input. This "autofocusing"" method solves the Marchenko equation and, subsequently, the 1D inverse scattering problem. Broggini and Snieder [4] show that the focusing wave field and its response can be combined in a specific way to yield the 1D medium's Green's function, with its virtual source at the focal point inside the medium.

The question arises whether the discussed concepts of single-sided Marchenko inverse scattering, autofocusing, Green's function retrieval, and their mutual relations [4] can be extended from one to three dimensions. A 3D extension of the Marchenko equation is the so-called Newton-Marchenko (NM) equation [5,6]. 3D inverse scattering based on the NM equation requires omnidirectional reflection and transmission measurements. Hence, despite the fact that the NM equation is very useful for this class of inverse problems, it is not applicable to single-sided reflection measurements. Prada et al. [7] pioneered 3D single-sided autofocusing, but their method is limited to focusing waves onto the strongest scatterers and does not account for multiple scattering.

We derive a $3 \mathrm{D}$ version of the Marchenko equation which relates the single-sided scalar reflection response
PACS numbers: 43.20.+g, 41.20.Jb, 43.60.+d, 91.30.-f

of a 3D inhomogeneous medium to the field inside the medium. Next, we briefly review a new 3D data-driven focusing method [8] and show that this method solves the 3D single-sided Marchenko equation. We also show that a specific combination of the 3D focusing wave field and its reflection response gives the 3D Green's function, with its virtual source at the focal point inside the medium. Unlike in other data-driven Green's function retrieval methods [9-12], no receiver is needed at the position of the virtual source.

Fundamental solutions. - In the derivation of the 1D Marchenko equation, so-called "fundamental solutions" of the source-free Schrödinger equation play an essential role [1]. For the situation of a localized potential $u(x)$, the fundamental solutions $f_{1}(x, t)$ and $f_{2}(x, t)$ of the Schrödinger equation are those solutions that reduce to impulsive outgoing waves for $x \rightarrow \infty$ and $x \rightarrow-\infty$, respectively. Here, we extend the fundamental solutions to 3D. We define the spatial coordinate vector $\mathbf{x}$ as $\mathbf{x}=$ $\left(\mathbf{x}_{H}, x_{3}\right)$, in which $\mathbf{x}_{H}=\left(x_{1}, x_{2}\right)$ is the horizontal coordinate vector and $x_{3}$ the vertical coordinate; the positive $x_{3}$ axis is pointing downward. Coordinates at a constant depth level $x_{3}=x_{3, i}$ are denoted as $\mathbf{x}_{i}=\left(\mathbf{x}_{H}, x_{3, i}\right)$ and time is denoted by $t$. Consider an inhomogeneous lossless medium between transparent boundaries $\partial \mathbb{D}_{0}$ and $\partial \mathbb{D}_{m}$ at depth levels $x_{3}=x_{3,0}$ and $x_{3}=x_{3, m}$, respectively. The domain enclosed by $\partial \mathbb{D}_{0}$ and $\partial \mathbb{D}_{m}$ is denoted as $\mathbb{D}$. The upper halfspace $x_{3}<x_{3,0}$ and the lower half-space $x_{3}>x_{3, m}$ are homogeneous. A fundamental solution $f_{1}(\mathbf{x}, t)$ of the scalar wave equation consists in the upper half-space of a downgoing field $f_{1}^{+}(\mathbf{x}, t)$ and an upgoing field $f_{1}^{-}(\mathbf{x}, t)$, with $f_{1}^{+}(\mathbf{x}, t)$ shaped such that $f_{1}(\mathbf{x}, t)$ focuses at $\mathbf{x}_{m}^{\prime}=$ $\left(\mathbf{x}_{H}^{\prime}, x_{3, m}\right)$ at $t=0$, and continues as an impulsive diverging downgoing field $f_{1}^{+}(\mathbf{x}, t)$ into the lower half-space. The focal point $\mathbf{x}_{m}^{\prime}$ is a variable at $\partial \mathbb{D}_{m}$ and will from here onward be included in the argument list; hence, $f_{1}(\mathbf{x}, t)$ becomes $f_{1}\left(\mathbf{x}, \mathbf{x}_{m}^{\prime}, t\right)$ [Fig. 1(a)]. Similarly, the 
fundamental solution $f_{2}\left(\mathbf{x}, \mathbf{x}_{0}^{\prime \prime}, t\right)$ focuses at $\mathbf{x}_{0}^{\prime \prime}=\left(\mathbf{x}_{H}^{\prime \prime}, x_{3,0}\right)$ at $t=0$, and continues as an impulsive diverging upgoing field $f_{2}^{-}\left(\mathbf{x}, \mathbf{x}_{0}^{\prime \prime}, t\right)$ into the upper half-space [Fig. 1(b)]. The temporal Fourier transform of a time-dependent function $u(t)$ is defined as $u(\omega)=\int_{-\infty}^{\infty} u(t) \exp (i \omega t) d t$, where $\omega$ is the angular frequency and $i$ the imaginary unit. To keep the notation simple, the same symbol is used for timeand frequency-domain functions (here $u$ ). In the frequency domain, the aforementioned focusing conditions for $f_{1}$ and $f_{2}$ are $f_{1}\left(\mathbf{x}_{m}, \mathbf{x}_{m}^{\prime}, \omega\right)=\delta\left(\mathbf{x}_{H}-\mathbf{x}_{H}^{\prime}\right)$ and $f_{2}\left(\mathbf{x}_{0}, \mathbf{x}_{0}^{\prime \prime}, \omega\right)=$ $\delta\left(\mathbf{x}_{H}-\mathbf{x}_{H}^{\prime \prime}\right)$. Throughout this paper we ignore evanescent waves; hence, head waves, turning waves, etc. are excluded from the following analysis. Moreover, $\delta\left(\mathbf{x}_{H}\right)$ should be interpreted as a spatially bandlimited delta function (because it lacks the wave number components of the evanescent field).

The two fundamental solutions are mutually related. The relation can be rigorously derived from reciprocity theorems for flux-normalized downgoing and upgoing wave fields [11]. Here we present a short, more intuitive,

(a)
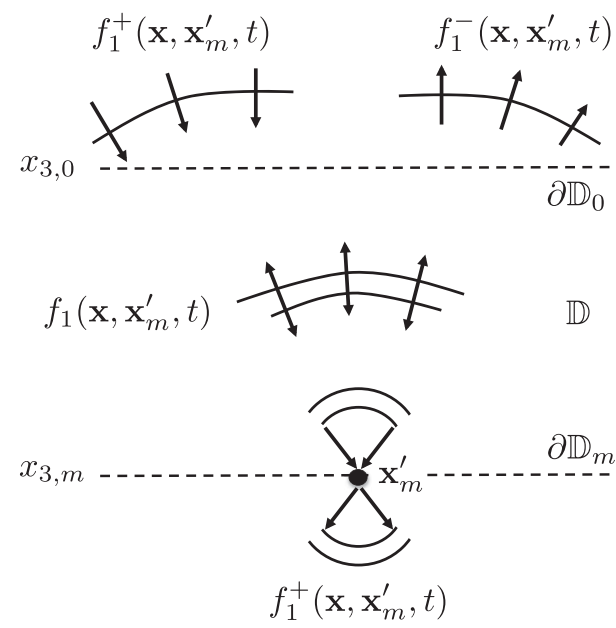

(b)
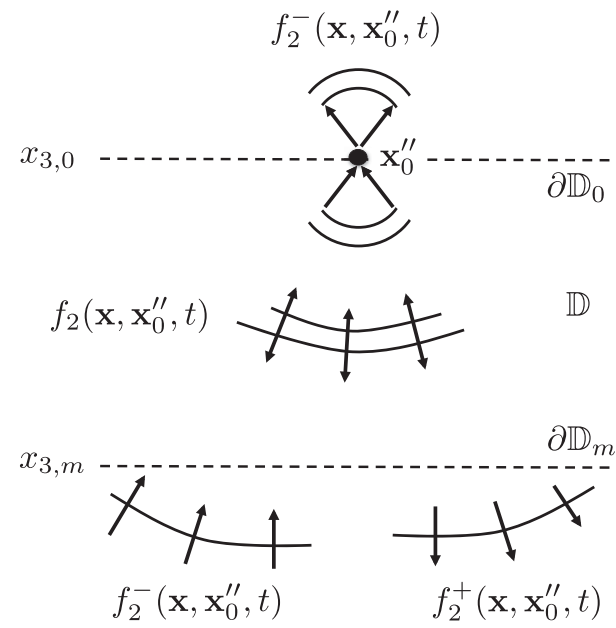

FIG. 1. Fundamental solutions of the 3D wave equation. derivation. In the upper half-space the upgoing field $f_{1}^{-}$ can be seen as the response to a distribution of Huygens sources along $\partial \mathbb{D}_{0}$, weighted by $f_{1}^{-}$at $\partial \mathbb{D}_{0}$. Since $f_{2}$ focuses at $\partial \mathbb{D}_{0}$ [Fig. 1(b)] and emits impulsive upward propagating waves into the upper half-space, $f_{2}$ at $\partial \mathbb{D}_{0}$ provides the Huygens sources. Similarly, the downgoing field $f_{1}^{+}$in the upper half-space can be seen as the acausal response to time-reversed Huygens sources (or Huygens sinks) $f_{2}^{*}$, weighted by $f_{1}^{+}$at $\partial \mathbb{D}_{0}$ (the asterisk denotes complex conjugation). Hence, for $f_{1}=f_{1}^{+}+f_{1}^{-}$we have the following in the upper half-space:

$$
\begin{aligned}
f_{1}\left(\mathbf{x}, \mathbf{x}_{m}^{\prime}, \omega\right)= & \int_{\partial \mathbb{D}_{0}} f_{2}\left(\mathbf{x}, \mathbf{x}_{0}^{\prime \prime}, \omega\right) f_{1}^{-}\left(\mathbf{x}_{0}^{\prime \prime}, \mathbf{x}_{m}^{\prime}, \omega\right) d \mathbf{x}_{0}^{\prime \prime} \\
& +\int_{\partial \mathbb{D}_{0}} f_{2}^{*}\left(\mathbf{x}, \mathbf{x}_{0}^{\prime \prime}, \omega\right) f_{1}^{+}\left(\mathbf{x}_{0}^{\prime \prime}, \mathbf{x}_{m}^{\prime}, \omega\right) d \mathbf{x}_{0}^{\prime \prime} .
\end{aligned}
$$

Recall that $\mathbf{x}_{0}^{\prime \prime}=\left(\mathbf{x}_{H}^{\prime \prime}, x_{3,0}\right)$; hence, the integration takes place at $\partial \mathbb{D}_{0}$ along the horizontal coordinate vector $\mathbf{x}_{H}^{\prime \prime}$. Since $f_{1}$ and $f_{2}$ are solutions of the one-and-the-same source-free wave equation for all $\mathbf{x} \in \mathbb{R}^{3}$, Eq. (1) not only holds in the upper half-space but throughout space. In a similar way, $f_{2}$ can be expressed in terms of $f_{1}$ and $f_{1}^{*}$.

The downgoing and upgoing constituents of $f_{1}$ at $\partial \mathbb{D}_{0}$ are related via the reflection response $R\left(\mathbf{x}_{0}, \mathbf{x}_{0}^{\prime \prime}, \omega\right)$ of the inhomogeneous medium in $\mathbb{D}$, according to

$f_{1}^{-}\left(\mathbf{x}_{0}, \mathbf{x}_{m}^{\prime}, \omega\right)=\int_{\partial \mathbb{D}_{0}} R\left(\mathbf{x}_{0}, \mathbf{x}_{0}^{\prime \prime}, \omega\right) f_{1}^{+}\left(\mathbf{x}_{0}^{\prime \prime}, \mathbf{x}_{m}^{\prime}, \omega\right) d \mathbf{x}_{0}^{\prime \prime}$.

Similarly, the downgoing field at $\partial \mathbb{D}_{m}$ is related to the downgoing field at $\partial \mathbb{D}_{0}$ via the transmission response $T\left(\mathbf{x}_{m}, \mathbf{x}_{0}^{\prime \prime}, \omega\right)$. Because the downgoing wave field focuses at $\mathbf{x}_{m}^{\prime}=\left(\mathbf{x}_{H}^{\prime}, x_{3, m}\right)$, this gives

$$
\delta\left(\mathbf{x}_{H}-\mathbf{x}_{H}^{\prime}\right)=\int_{\partial \mathbb{D}_{0}} T\left(\mathbf{x}_{m}, \mathbf{x}_{0}^{\prime \prime}, \omega\right) f_{1}^{+}\left(\mathbf{x}_{0}^{\prime \prime}, \mathbf{x}_{m}^{\prime}, \omega\right) d \mathbf{x}_{0}^{\prime \prime} .
$$

We introduce the inverse of $f_{1}^{+}\left(\mathbf{x}_{0}, \mathbf{x}_{m}^{\prime}, \omega\right)$ via $\int_{\partial \mathbb{D}_{m}} f_{1}^{+}\left(\mathbf{x}_{0}, \mathbf{x}_{m}^{\prime}, \omega\right)\left\{f_{1}^{+}\left(\mathbf{x}_{0}^{\prime \prime}, \mathbf{x}_{m}^{\prime}, \omega\right)\right\}^{\text {inv }} d \mathbf{x}_{m}^{\prime}=\delta\left(\mathbf{x}_{H}-\mathbf{x}_{H}^{\prime \prime}\right)$. Applying this inverse to both sides of Eq. (3) gives $\left\{f_{1}^{+}\left(\mathbf{x}_{0}^{\prime \prime}, \mathbf{x}_{m}, \omega\right)\right\}^{\mathrm{inv}}=T\left(\mathbf{x}_{m}, \mathbf{x}_{0}^{\prime \prime}, \omega\right)$. Applying $T\left(\mathbf{x}_{m}^{\prime}, \mathbf{x}_{0}^{\prime \prime}, \omega\right)$ to both sides of Eq. (1) gives, using Eq. (2),

$$
\begin{aligned}
\int_{\partial \mathbb{D}_{m}} & f_{1}\left(\mathbf{x}, \mathbf{x}_{m}^{\prime}, \omega\right) T\left(\mathbf{x}_{m}^{\prime}, \mathbf{x}_{0}^{\prime \prime}, \omega\right) d \mathbf{x}_{m}^{\prime} \\
\quad= & \int_{\partial \mathbb{D}_{0}} f_{2}\left(\mathbf{x}, \mathbf{x}_{0}^{\prime}, \omega\right) R\left(\mathbf{x}_{0}^{\prime}, \mathbf{x}_{0}^{\prime \prime}, \omega\right) d \mathbf{x}_{0}^{\prime}+f_{2}^{*}\left(\mathbf{x}, \mathbf{x}_{0}^{\prime \prime}, \omega\right) .
\end{aligned}
$$

In the following we show that the left-hand side of Eq. (4) is equal to the Green's function $G\left(\mathbf{x}, \mathbf{x}_{0}^{\prime \prime}, \omega\right)$ and we use the right-hand side to derive the 3D Marchenko equation.

Green's function.-We define an internal boundary $\partial \mathbb{D}_{i}$ at depth level $x_{3, i}$, anywhere between $x_{3,0}$ and $x_{3, m}$, and analyze the Green's function $G\left(\mathbf{x}_{i}, \mathbf{x}_{0}^{\prime \prime}, \omega\right)$. The downgoing and upgoing constituents, $G^{+}$and $G^{-}$, are related via [13] 
$G^{-}\left(\mathbf{x}_{i}^{\prime}, \mathbf{x}_{0}^{\prime \prime}, \omega\right)=\int_{\partial \mathbb{D}_{i}} R\left(\mathbf{x}_{i}^{\prime}, \mathbf{x}_{i}, \omega\right) G^{+}\left(\mathbf{x}_{i}, \mathbf{x}_{0}^{\prime \prime}, \omega\right) d \mathbf{x}_{i}$.

This equation resembles Eq. (2), but because $\partial \mathbb{D}_{i}$ is an internal boundary it is less trivial. $R\left(\mathbf{x}_{i}^{\prime}, \mathbf{x}_{i}, \omega\right)$ is the reflection response of a truncated medium (consisting of the actual medium below $\partial \mathbb{D}_{i}$ and a scattering-free half-space above $\partial \mathbb{D}_{i}$ ), whereas the Green's function is defined in the actual medium. Using a similar derivation as in Ref. [13], we also obtain

$\int_{\partial \mathbb{D}_{i}} T\left(\mathbf{x}_{m}^{\prime}, \mathbf{x}_{i}, \omega\right) G^{+}\left(\mathbf{x}_{i}, \mathbf{x}_{0}^{\prime \prime}, \omega\right) d \mathbf{x}_{i}=T\left(\mathbf{x}_{m}^{\prime}, \mathbf{x}_{0}^{\prime \prime}, \omega\right)$,

where $T\left(\mathbf{x}_{m}^{\prime}, \mathbf{x}_{i}, \omega\right)$ is the transmission response of the truncated medium. Similar as above we have $\left\{f_{1}^{+}\left(\mathbf{x}_{i}, \mathbf{x}_{m}^{\prime}, \omega\right)\right\}^{\text {inv }}=T\left(\mathbf{x}_{m}^{\prime}, \mathbf{x}_{i}, \omega\right)$. Substituting this in the left-hand side of Eq. (6) and applying $f_{1}^{+}$to both sides gives

$G^{+}\left(\mathbf{x}_{i}, \mathbf{x}_{0}^{\prime \prime}, \omega\right)=\int_{\partial \mathbb{D}_{m}} f_{1}^{+}\left(\mathbf{x}_{i}, \mathbf{x}_{m}^{\prime}, \omega\right) T\left(\mathbf{x}_{m}^{\prime}, \mathbf{x}_{0}^{\prime \prime}, \omega\right) d \mathbf{x}_{m}^{\prime}$.

Applying the reflection response $R$ to both sides of Eq. (7) gives, using Eq. (5) for $G^{ \pm}$and $f_{1}^{ \pm}$,

$G^{-}\left(\mathbf{x}_{i}, \mathbf{x}_{0}^{\prime \prime}, \omega\right)=\int_{\partial \mathbb{D}_{m}} f_{1}^{-}\left(\mathbf{x}_{i}, \mathbf{x}_{m}^{\prime}, \omega\right) T\left(\mathbf{x}_{m}^{\prime}, \mathbf{x}_{0}^{\prime \prime}, \omega\right) d \mathbf{x}_{m}^{\prime}$.

Summing Eqs. (7) and (8) and dropping the subscript $i$ confirms that the left-hand side of Eq. (4) is equal to the Green's function $G\left(\mathbf{x}, \mathbf{x}_{0}^{\prime \prime}, \omega\right)$ for $\mathbf{x} \in \mathbb{D}$.

$3 D$ Marchenko equation.-We transform Eq. (4), with the left-hand side replaced by $G\left(\mathbf{x}, \mathbf{x}_{0}^{\prime \prime}, \omega\right)$, to the time domain, which gives

$$
\begin{aligned}
G\left(\mathbf{x}, \mathbf{x}_{0}^{\prime \prime}, t\right)= & \int_{\partial \mathbb{D}_{0}} d \mathbf{x}_{0}^{\prime} \int_{-\infty}^{\infty} f_{2}\left(\mathbf{x}, \mathbf{x}_{0}^{\prime}, t^{\prime}\right) R\left(\mathbf{x}_{0}^{\prime}, \mathbf{x}_{0}^{\prime \prime}, t-t^{\prime}\right) d t^{\prime} \\
& +f_{2}\left(\mathbf{x}, \mathbf{x}_{0}^{\prime \prime},-t\right) .
\end{aligned}
$$

Let $t_{d}\left(\mathbf{x}, \mathbf{x}_{0}^{\prime \prime}\right)$ denote the travel time of the first arrival between $\mathbf{x}_{0}^{\prime \prime} \in \partial \mathbb{D}_{0}$ and $\mathbf{x} \in \mathbb{D}$. By evaluating Eq. (9) only for $t<t_{d}\left(\mathbf{x}, \mathbf{x}_{0}^{\prime \prime}\right)$, the left-hand side can be replaced by zero. Equation (9) (with 0 on the left-hand side) only constrains $f_{2}$ up to a multiplicative constant and therefore an ansatz will be made for the form of this function. In the $1 \mathrm{D}$ derivation [1], $f_{2}(x, t)$ is defined as a delta pulse traveling in the negative $x$ direction, followed by a scattering coda caused by the potential $u(x)$. Moreover, the incident field is shaped such that the scattering coda vanishes beyond the scattering region, leaving only the delta pulse for $x \rightarrow-\infty$. Analogous to the 1D situation, the ansatz for $f_{2}\left(\mathbf{x}, \mathbf{x}_{0}^{\prime \prime}, t\right)$ is a superposition of a direct wave and a scattering coda, according to

$$
\begin{aligned}
f_{2}\left(\mathbf{x}, \mathbf{x}_{0}^{\prime \prime}, t\right)= & \left\{T_{d}\left(\mathbf{x}, \mathbf{x}_{0}^{\prime \prime}, t\right)\right\}^{\text {inv }} \\
& +\theta\left(t+t_{d}\left(\mathbf{x}, \mathbf{x}_{0}^{\prime \prime}\right)\right) M\left(\mathbf{x}, \mathbf{x}_{0}^{\prime \prime}, t\right) .
\end{aligned}
$$

Here $\left\{T_{d}\left(\mathbf{x}, \mathbf{x}_{0}^{\prime \prime}, t\right)\right\}^{\text {inv }}$ is the inverse of the direct arrival of the transmission response, which focuses at $\mathbf{x}_{0}^{\prime \prime}$; its travel time is $-t_{d}\left(\mathbf{x}, \mathbf{x}_{0}^{\prime \prime}\right)$. In practice, it will often suffice to approximate $\left\{T_{d}\left(\mathbf{x}, \mathbf{x}_{0}^{\prime \prime}, t\right)\right\}^{\text {inv }}$ by the time reversal $T_{d}\left(\mathbf{x}, \mathbf{x}_{0}^{\prime \prime},-t\right)$, or even by $\delta\left(t+t_{d}\left(\mathbf{x}, \mathbf{x}_{0}^{\prime \prime}\right)\right) . M\left(\mathbf{x}, \mathbf{x}_{0}^{\prime \prime}, t\right)$ is the coda following the direct arrival. It is the result of scattering taking place in the inhomogeneous medium in $\mathbb{D}$. The coda is assumed to be causal, i.e., $M\left(\mathbf{x}, \mathbf{x}_{0}^{\prime \prime}, t\right)=0$ for $t<-t_{d}\left(\mathbf{x}, \mathbf{x}_{0}^{\prime \prime}\right)$. This causality is expressed by multiplying the coda with the Heaviside function $\theta(t+$ $\left.t_{d}\left(\mathbf{x}, \mathbf{x}_{0}^{\prime \prime}\right)\right)$ in Eq. (10). Note that the ansatz limits the validity of what follows to configurations for which the ansatz holds true. For example, it holds in layered media with moderately curved interfaces as long as $\left|\mathbf{x}_{H}-\mathbf{x}_{H}^{\prime \prime}\right|$ is not too large (to avoid the occurrence of turning waves, head waves, etc.). The conditions underlying the ansatz need further investigation, which is beyond the scope of this paper. Substituting Eq. (10) into (9) (with 0 on the left-hand side) yields

$$
\begin{aligned}
0= & \int_{\partial \mathbb{D}_{0}} d \mathbf{x}_{0}^{\prime} \int_{-\infty}^{\infty}\left\{T_{d}\left(\mathbf{x}, \mathbf{x}_{0}^{\prime}, t^{\prime}\right)\right\}^{\text {inv }} R\left(\mathbf{x}_{0}^{\prime}, \mathbf{x}_{0}^{\prime \prime}, t-t^{\prime}\right) d t^{\prime} \\
& +\int_{\partial \mathbb{D}_{0}} d \mathbf{x}_{0}^{\prime} \int_{-t_{d}\left(\mathbf{x}, \mathbf{x}_{0}^{\prime}\right)}^{\infty} M\left(\mathbf{x}, \mathbf{x}_{0}^{\prime}, t^{\prime}\right) R\left(\mathbf{x}_{0}^{\prime}, \mathbf{x}_{0}^{\prime \prime}, t-t^{\prime}\right) d t^{\prime} \\
& +M\left(\mathbf{x}, \mathbf{x}_{0}^{\prime \prime},-t\right)
\end{aligned}
$$

with $\mathbf{x} \in \mathbb{D}$ and $t<t_{d}\left(\mathbf{x}, \mathbf{x}_{0}^{\prime \prime}\right)$. This is the $3 \mathrm{D}$ single-sided Marchenko equation. Next we show that it can be solved with an iterative 3D data-driven focusing scheme.

$3 D$ data-driven focusing. - Inspired by the work of Rose [3], the authors proposed an iterative scheme to design a downgoing wave field $p^{+}\left(\mathbf{x}_{0}, t\right)$ at $\partial \mathbb{D}_{0}$ that focuses at $t=0$ at a focal point $\mathbf{x}_{F} \in \mathbb{D}$ (and at $\mathbf{x}_{F}$ only) [8]. The initial estimate $p_{0}^{+}\left(\mathbf{x}_{0}, t\right)$ is defined as $p_{0}^{+}\left(\mathbf{x}_{0}, t\right)=$ $\left\{T_{d}\left(\mathbf{x}_{0}, \mathbf{x}_{F}, t\right)\right\}^{\text {inv }}$. When emitted from $\partial \mathbb{D}_{0}$ into the inhomogeneous medium, this field not only focuses at $\mathbf{x}_{F}$, but it also causes ghost images at $t=0$. These ghost images can be canceled by updating the incident field, which causes new ghost images, which again need to be canceled, etc. The following iterative scheme accomplishes this task [8]:

$$
\begin{aligned}
p_{k}^{+}\left(\mathbf{x}_{0}^{\prime}, \mathbf{x}_{F}, t\right)= & \left\{T_{d}\left(\mathbf{x}_{0}^{\prime}, \mathbf{x}_{F}, t\right)\right\}^{\text {inv }} \\
& -\theta\left(t+t_{d}\left(\mathbf{x}_{0}^{\prime}, \mathbf{x}_{F}\right)\right) p_{k-1}^{-}\left(\mathbf{x}_{0}^{\prime}, \mathbf{x}_{F},-t\right),
\end{aligned}
$$

$$
\begin{aligned}
& p_{k}^{-}\left(\mathbf{x}_{0}^{\prime \prime}, \mathbf{x}_{F}, t\right) \\
& \quad=\int_{\partial \mathbb{D}_{0}} d \mathbf{x}_{0}^{\prime} \int_{-\infty}^{\infty} R\left(\mathbf{x}_{0}^{\prime \prime}, \mathbf{x}_{0}^{\prime}, t-t^{\prime}\right) p_{k}^{+}\left(\mathbf{x}_{0}^{\prime}, \mathbf{x}_{F}, t^{\prime}\right) d t^{\prime}
\end{aligned}
$$

Here $p_{k}^{+}\left(\mathbf{x}_{0}^{\prime}, \mathbf{x}_{F}, t\right)$ is the $k$ th iteration of the downgoing wave field, intended to focus at $\mathbf{x}_{F}$, whereas $p_{k}^{-}\left(\mathbf{x}_{0}^{\prime \prime}, \mathbf{x}_{F}, t\right)$ is its upgoing reflection response. The scheme starts for $k=0$, with $p_{-1}^{-}\left(\mathbf{x}_{0}^{\prime}, \mathbf{x}_{F},-t\right)=0$. Unlike the $1 \mathrm{D}$ scheme of Rose, which only needs the reflection response as input, 
this 3D scheme requires the reflection response $R\left(\mathbf{x}_{0}^{\prime \prime}, \mathbf{x}_{0}^{\prime}, t\right)$ and the direct transmission response $T_{d}\left(\mathbf{x}_{0}^{\prime}, \mathbf{x}_{F}, t\right)$. The reflection response is measured at the boundary $\partial \mathbb{D}_{0}$. The transmission response mainly requires an estimate of the direct arrival time $t_{d}\left(\mathbf{x}_{0}^{\prime}, \mathbf{x}_{F}\right)$, for which no detailed information about the medium is required: a smooth background model suffices to compute the direct transmission response $T_{d}\left(\mathbf{x}_{0}^{\prime}, \mathbf{x}_{F}, t\right)$. Note that estimating the background model is state-of-the-art methodology in geophysical imaging [14]. All information about the scattering properties of the medium comes from the measured reflection response. This is why we call the scheme of Eqs. (12) and (13) and "data-driven focusing."

Assuming the scheme converges, the final result is denoted by dropping the subscripts $k-1$ and $k$. Substituting Eq. (12) into Eq. (13), using source-receiver reciprocity for the reflection and transmission responses, gives for $t<t_{d}\left(\mathbf{x}_{F}, \mathbf{x}_{0}^{\prime \prime}\right)$ the 3D Marchenko equation, (11), with $M\left(\mathbf{x}_{F}, \mathbf{x}_{0}^{\prime \prime},-t\right)=-p^{-}\left(\mathbf{x}_{0}^{\prime \prime}, \mathbf{x}_{F}, t\right)$. Hence, the iterative 3D data-driven focusing scheme of Eqs. (12) and (13) solves the 3D Marchenko equation.

$3 D$ Green's function retrieval.-Here we generalize the approach of Broggini and Snieder [4] to retrieve the 3D Green's function. A comparison of Eq. (10) with Eq. (12) (without the subscripts $k-1$ and $k$ ) gives $f_{2}\left(\mathbf{x}_{F}, \mathbf{x}_{0}^{\prime}, t\right)=$ $p^{+}\left(\mathbf{x}_{0}^{\prime}, \mathbf{x}_{F}, t\right)$. Substituting this into Eq. (9), using Eq. (13) and source-receiver reciprocity for the Green's function, gives (dropping the primes)

$$
G\left(\mathbf{x}_{0}, \mathbf{x}_{F}, t\right)=p^{+}\left(\mathbf{x}_{0}, \mathbf{x}_{F},-t\right)+p^{-}\left(\mathbf{x}_{0}, \mathbf{x}_{F}, t\right) .
$$

This shows that a combination of the focusing wave field $p^{+}$and its response $p^{-}$yields the Green's function with its virtual source at the position of the focal point inside the medium. Unlike in other Green's function retrieval methods [9-12], no physical receiver is required at the position of the virtual source. The method is illustrated with a $2 \mathrm{D}$ numerical example. Figure 2(a) shows an inhomogeneous medium (the colors represent the propagation velocity $c$ ). The yellow dot represents the virtual source position $\mathbf{x}_{F}$ and the yellow triangles represent 23 receiver positions $\mathbf{x}_{0}$ at the surface. Figure 2(b) shows the direct transmission response $T_{d}\left(\mathbf{x}_{0}, \mathbf{x}_{F}, t\right)$ for all 23 receiver positions, modeled in a smoothed version of the medium. This direct field is used in the iterative scheme of Eqs. (12) and (13), together with the reflection response at the surface (not shown). Figure 2(c) shows the Green's function $G\left(\mathbf{x}_{0}, \mathbf{x}_{F}, t\right)$ obtained from Eq. (14) (black dashed traces), overlain on the directly modeled Green's function (red traces). All traces have been multiplied by $\exp (2 t)$ to emphasize the scattering coda. Note that this coda is very well recovered.

Concluding remarks. - We have derived a 3D version of the Marchenko equation, which relates the single-sided 3D reflection response of an inhomogeneous medium to a field inside the medium. In the derivation we assumed scalar
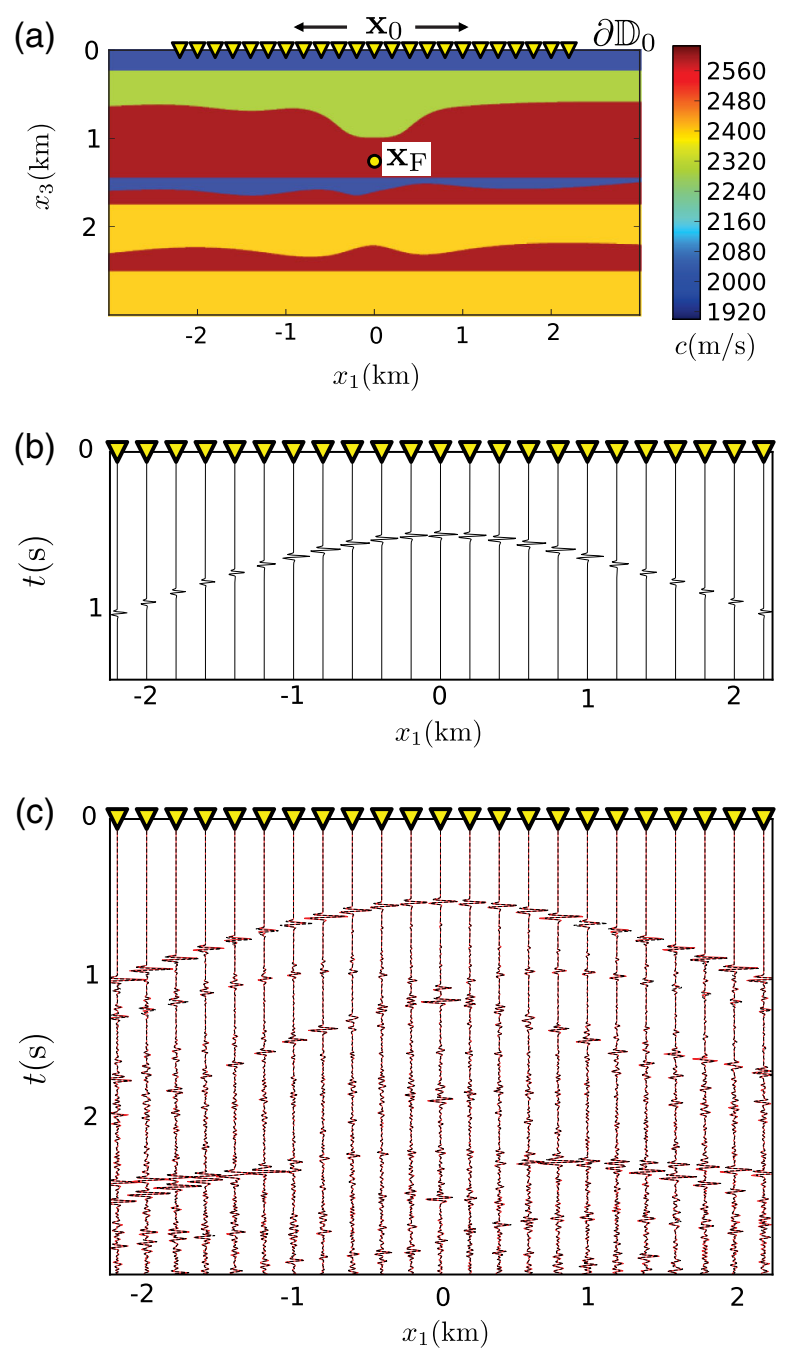

FIG. 2 (color online). Numerical example.

waves in a lossless medium, ignored evanescent waves, and further assumed that the scattering coda of the fundamental solution follows the inverse of the direct arrival of the transmission response. These conditions imply some restrictions that need further investigation. For those situations in which the conditions are fulfilled, we showed that the 3D single-sided Marchenko equation is solved by an iterative data-driven focusing scheme. This scheme requires the $3 \mathrm{D}$ reflection response at one side of the medium and an estimate of the direct arrival of the transmission response. It is, in fact, through the arrival time of direct arrivals that one specifies the location of the focal point. We also showed that a combination of the focusing wave field and its reflection response gives the 3D Green's function with its virtual source located at the focal point. Because no physical receiver is needed at the position of the focal point, the focal point can be chosen anywhere inside the medium. This gives the possibility to obtain Green's functions with virtual sources throughout the medium, which can be used for the imaging of objects that are accessible from one side only. The methodology will be of 
particular interest for situations in which the target image is blurred by multiple scattering. The next challenge is to extend the method to vector wave fields. We foresee applications in many areas, ranging from nondestructive inspection of construction materials to seismological reflection imaging and monitoring of structures and processes in the Earth's interior.

We thank Jan Thorbecke and Joost van der Neut for their contributiuons to the numerical experiment.

*c.p.a.wapenaar@tudelft.nl

[1] G. L. Lamb, Elements of Soliton Theory (John Wiley and Sons, Inc., New York, 1980).

[2] R. Burridge, Wave Motion 2, 305 (1980).

[3] J. H. Rose, Phys. Rev. A 65, 012707 (2001).

[4] F. Broggini and R. Snieder, Eur. J. Phys. 33, 593 (2012).
[5] R. G. Newton, J. Math. Phys. (N.Y.) 23, 594 (1982).

[6] D. E. Budreck and J.H. Rose, Inverse Probl. 6, 331 (1990).

[7] C. Prada, F. Wu, and M. Fink, J. Acoust. Soc. Am. 90, 1119 (1991).

[8] K. Wapenaar, F. Broggini, and R. Snieder, Geophys. J. Int. 190, 1020 (2012).

[9] R. L. Weaver and O. I. Lobkis, Phys. Rev. Lett. 87, 134301 (2001).

[10] M. Campillo and A. Paul, Science 299, 547 (2003).

[11] K. Wapenaar, J. Thorbecke, and D. Draganov, Geophys. J. Int. 156, 179 (2004).

[12] G. T. Schuster, Seismic Interferometry (Cambridge University Press, Cambridge, 2009).

[13] K. Wapenaar, E. Slob, and R. Snieder, Geophysical Prospecting 56, 419 (2008).

[14] W.S. Harlan, R. T. Langan, and T. Nemeth, Geophysics 73, VE1 (2008). 